von Jodkalium ist für das Gelingen der Trennung unbedingt erforderlich. Nachdem die Lösung bis zur richtigen Concentration eingedampft worden, fällt man mit Gallussäure.

Hat man die vorerwähnten Operationen alle richtig ausgeführt, so setzt sich das gallussaure Antimonoxyd sehr rasch zu Boden. Sollte dies nicht der Fall sein, so ist die Trennung als nicht gelungen zu verwerfen, der Niederschlag enthält dann immer auch grössere oder kleinere Quantitäten von Zinnoxyd.

Die Trennung des Antimons von Zinn und Arsen wird in derselben Weise ausgeführt, wie die vom Zinr allein, sie gelingt jedoch noch schwieriger. Den Grund dafür hat der Verfasser bis jetzt noch nicht ermitteln können.

\title{
Ueber die Bestimmung des Arsens als pyroarsensaure Magnesia
} hat L. F. W o od ${ }^{*}$ ) seine Erfahrungen mitgetheilt.

Zunächst gibt er einen historischen Ueberblick über die Entwickelung der Methode and erwähnt namentlich derjenigen Arbeiten, durch welche dargethan wurde, dass die arsensaure Ammon-Magnesia durch sorgfältiges Erhitzen ohne Verlust in pyroarsensaure Magnesia übergeführt und als solche gewogen werden kann. ${ }^{* *}$ )

Als Fällungsmittel für die Arsensäure schlägt der Verfasser eine Lösung von reinem Chlormagnesium in Alkohol von etwa 85 Procent vor. Gewöhnlich bedient er sich einer Lösung, welche etwa $100 \mathrm{Grm} . \mathrm{Mg} \mathrm{Cl}$ im Liter enthält.

Um sich von der Anwendbarkeit dieses Fällungsmittels zu überzengen, stellte $\mathrm{Wood}$ die nachstehend mitgetheilten Versuche an. Zn jeder Analyse wurden 0,3000 Grm. arsenige Säure angewandt, in einer 2 Grm. kohlensaures Natron enthaltenden Lösung aufgelöst und in bekannter Weise mit Chlorgas oxydirt. Die Lösung wurde dann mit Salzsäure angesäuert, etwa $2 \mathrm{Grm}$. festes Chlorammonium zugefügt und dann wässeriges Ammoniak in bedeutendem Ueberschuss. Hierauf setzte man von der alkoholischen Chlormagnesiumlösung zu, so lange noch ein Niederschlag entstand, dann noch 1,5 CC. mehr und liess über Nacht stehen. Es wurde jetzt eine Quantität 85procentigen Alkohols zugefügt, die etwa dem halben Volum der Flüssigkeit gleichkam, dann wurde der Nieder-

*) Amer. Journ. of science and arts [3 ser.] 6, 368 .

**) Ein ähnlicher historischer Ueberblick über diese Sache findet sich in dieser Zeitschrift 14, 96, auf welchen wir dieserhalb verweisen. 
schlag auf ein gewogenes Filter abfiltrirt und mit einer Mischung von 2 Thln. Alkohol, 1 Thl. starker Ammoniakflüssigkeit und 3 Thln. Wasser ausgewaschen.

Jedesmal wenn ein Filter gewogen wurde, legte man auf die andere Wagschale ein Filter von demselben Durchmesser um annäherndes Gleichgewicht herzustellen.

Da ein Verlust unvermeidlich ist, wenn man das Filter mit daranhängender arsensaurer Ammon-Magnesia verbrennt und da andererseits das vollständige Trocknen einer grossen Menge des Niederschlages bei $100^{0}$ sehr mühsam ist, ${ }^{*}$ ) so wurde nur die kleine Menge des Niederschlages, welche sich nicht leicht vom Filter trennen liess sammt diesem bei $100^{\circ}$ bis $\mathrm{zu}$ constantem Gewicht getrocknet und gewogen. Die Hauptmenge des Niederschlages wurde in einen Porcellantiegel gebracht, mit 3 bis 4 Tropfen starker Salpetersäure befeuchtet, das Ganze in einen grösseren Tiegel gesetzt und nun erst etwa 15 Minuten lang bei einer langsam und allmählich gesteigerten Temperatur erhitzt, um die Salpetersäure und das Ammon zu verjagen. Das Einsetzen des kleineren Tiegels in einen grösseren geschah, um einen Verlust durch Verspritzen $\mathrm{zu}$ vermeiden und um reducirende Gase abzuhalten. Gleichzeitig wurde dadurch der Tiegel vor Abnutzung geschützt, so dass er nach Ausführung von sieben Analysen keine Differenz im Gewicht gegenüber der ersten Wägung zeigte.

Schliesslich wurden die Tiegel mehrmals je 5 Minuten lang bis zu coñstantem Gewicht über dem Gebläse erhitzt. Da die Zersetzungsproducte des salpetersauren Ammons oxydirend wirken, so ist kein Grand vorhanden, dabei eine Reduction der Arsensäure zu befürchten und aus den folgenden Resultaten scheint hervorzugehen, dass ein Verlust durch Verflüchtigung nicht stattfindet.

$\mathrm{Zu}$ sämmtlichen Analysen wurden, wie schon oben erwähnt, 0,3000 Grm. $\mathrm{As} \mathrm{O}_{3}$ genommen. Das Gewicht der gefundenen Arsensäure wurde berechnet aus dem Gewicht der pyroarsensauren Magnesia im Tiegel und dem der wasserhaltigen arsensauren Ammon-Magnesia auf dem Filter.

I. Filtrat $137 \mathrm{CC}$. Waschwasser $54 \mathrm{CC}$.

$2 \mathrm{MgO}, \mathrm{AsO}_{5}$. . . . . = $=0,4637 \mathrm{Grm}$.

$2 \mathrm{MgO}, \mathrm{N} \mathrm{H}_{4} \mathrm{O}, \mathrm{As}_{5} \mathrm{O}_{5}+\mathrm{aq}$. $=0,0076 \approx$

Daraus berechnet sich $\mathrm{As} \mathrm{O}_{3}=0,30013$ «

*) Vergl. Puller diese Zeitschr. 10, 63. 
II. Filtrat $146 \mathrm{CC}$. Waschwasser $50 \mathrm{CC}$.

$2 \mathrm{MgO}, \mathrm{As}_{5}$. . . . . $=0,4637 \mathrm{Grm}$.

$2 \mathrm{MgO}, \mathrm{NH}_{4} \mathrm{O}$, As $\mathrm{O}_{5}+\mathrm{aq} .=0,0076 \ll$

Daraus berechnet sich $\mathrm{As}_{3}=0,30013$ «

III. Filtrat $106 \mathrm{CC}$. Waschwasser $58 \mathrm{CC}$.

$2 \mathrm{MgO}, \mathrm{As}_{5}$. . . . . $=0,4576 \mathrm{Grm}$.

$2 \mathrm{MgO}, \mathrm{N} \mathrm{H}_{4} \mathrm{O}$, As $\mathrm{O}_{5}+\mathrm{aq} .=0,0134 \ll$

Daraus berechnet sich $\mathrm{As}_{3}=0,2992$ *

IV. Bei diesem Versuche liess man die Flüssigkeit nach dem Zusatz der alkoholischen Chlormagnesiumlösung und des Alkohols vier Tage lang stehen. Das Resultat zeigt, dass Magnesiahydrat oder kohlensaure Magnesia mit niedergefallen war.

$$
\begin{aligned}
& 2 \mathrm{MgO}, \mathrm{As}_{5} . . \quad . \quad=0,4962 \mathrm{Grm} . \\
& 2 \mathrm{MgO}, \mathrm{N} \mathrm{H}_{4} \mathrm{O}, \mathrm{As} \mathrm{O}_{5}+\mathrm{aq} .=0,0083 \\
& \text { Daraus berechnet sich } \mathrm{As} \mathrm{O}_{3}=0,3212
\end{aligned}
$$

Bei den folgenden vier Analysen wrurde, um diese Fehlerquelle zu vermeiden, der ursprünglich gebildete Nieảerschlag, nachdem er abfiltrirt worden war, in Salzsäure aufgelöst und durch Zufügen von überschüssiger Ammoniakfüssigkeit wieder ausgefällt; hierauf setzte man der Flüssigkeit noch die Hälfte ihres Volums Alkohol zu. Es war auf die Resultate ohne Einfluss, ob man den Niederschlag dann sofort oder nach 24ständigem Stehen abfiltrirte. Will man doppelte Fällung anwenden, so wird man bei der ersten Fällung den Alkohol am besten gleich zusetzen, anstatt vorher 24 Standen stehen zu lassen.

V. Erstes Filtrat 109 CC., zweites Filtrat 68 CC., Waschwasser $45 \mathrm{CC}$.

$$
\begin{aligned}
& 2 \mathrm{MgO} \text {, As } 0_{5} \text {. . . . . }=0,4630 \mathrm{Grm} \text {. } \\
& 2 \mathrm{MgO}, \mathrm{N} \mathrm{H}_{4} \mathrm{O}, \mathrm{As}_{5}+\text { aq. }=0,0068 \ll \\
& \text { Daraus berechnet sich } \mathrm{As}_{3}=0,2992 \text { « }
\end{aligned}
$$

VI. Erstes Filtrat $125 \mathrm{CC}$, , zweites Filtrat $64 \mathrm{CC}$., Waschwasser $40 \mathrm{CC}$.

$2 \mathrm{MgO}, \mathrm{As}_{5}$. . . . . $=0,4596 \mathrm{Grm}$.

$2 \mathrm{MgO}, \mathrm{N} \mathrm{H}_{4} \mathrm{O}, \mathrm{As} \mathrm{O}_{5}+\mathrm{aq}$. $=0,0124 \ll$

Daraus berechnet sich $\mathrm{AsO}_{3}=0,2999$ *

VII. Erstes Filtrat $165 \mathrm{CC}$., zweites Filtrat*), Waschwasser $45 \mathrm{CC}$.

$2 \mathrm{MgO}, \mathrm{As}_{5}$. . . . . $=0,4615 \mathrm{Grm}$.

$2 \mathrm{MgO}, \mathrm{N} \mathrm{H}_{4} \mathrm{O}, \mathrm{As} \mathrm{O}_{5}+$ aq. $=0,0099 \ll$

Daraus berechnet sich $\mathrm{As}_{3}=0,2999$ «

*) Im Original fehlt die Zahl. 
Zwei von den Filtraten und ein Waschwasser wurden auf Arsensäure geprüft und davon völlig frei befunden.

Zur Bestimmung der Schwefelsäure als schwefelsaurer Baryt. Die unangenehme Eigenschaft des schwefelsauren Barytes trotz aller Vorsicht häufig durchs Filter za gehen, ist jedem Chemiker genugsam bekannt. Da man diese Eigenthümlichlkeit des genannten Körpers einer ausserordentlichen Feinkörnigkeit zuschreiben muss, so versuchte Leo Liebermann*) den Niederschlag durch irgend ein Klebemittel grobkörniger zu machen und es gelang ihm dies mit Amylum. Versetzt man nämlich die Flüssigkeit, in der $\mathrm{BaO}, \mathrm{SO}_{3}$ gefällt wurde, mit einer kleinen Federmesserspitze Amylum and erwärmt 5-6 Minuten, indem man fortwährend mit einem Glasstabe umrührt, so lässt sich der schwefelsaure Baryt, gleichviel ob heiss oder kalt, ob abgesessen oder aufgerührt, bei richtiger Manipulation auch durch ordinäres Filtrirpapier, mit vollkommen klarem Filtrat trennen und mit kaltem Wasser waschen.

Wohl zu beachten ist jedoch, dass dies nicht gelingt, wenn der Flüssigkeit, in der die Schwefelsäure bestimmt werden soll, vor deren Ausfällung Amylum zugesetzt wird, namentlich, wenn die Flüssigkeit stark sauer ist.

Dieses Verfahren wurde sowohl von dem Verfasser, als auch auf dessen Veranlassung von mehreren Praktikanten versucht, fü sehr bequem und auch für quantitative Bestimmangen tauglich befunden. Noch sei erwähnt, dass mehrere Versuche ergaben, dass ein halber CC. von einem Wasser, das in 500 CC. 1 Grm. Amylum suspendirt enthält, also eine Menge von ungefähr 0,0002 Grm. Amylum genügt, um 0,5-1,0 Grm. $\mathrm{BaO}, \mathrm{SO}_{3}$ filtrirbar zu machen. $\mathrm{Zu}$ folgenden 3 Beleganalysen wurde immer eine kleine Federmesserspitze Amylum verwendet. Wie dieselben zeigen, ist die Reduction des schwefelsauren Baryts durch die Kohle des Amylums, beim Glühen des Niederschlages, von keiner wesentlichen Bedeutung.

I. 3,044 Grm. $\mathrm{FeO}, \mathrm{SO}_{3}+7 \mathrm{HO}$ gaben mit $\mathrm{BaCl}$ und Amylum $=$ $2,573^{\circ} \mathrm{Grm} . \mathrm{BaO}, \mathrm{SO}_{3}$. Demnach an Schwefelsäure:
gefunden $35,54 \mathrm{pCt}$.
berechnet 35,25 pCt.

II. 2,032 Grm. CuO, $\mathrm{SO}_{3}+5 \mathrm{HO}$ gaben mit $\mathrm{Ba} \mathrm{Cl}$ und Amylum $=$ 1,896 Grm. $\mathrm{BaO}, \mathrm{SO}_{3}$. Demnach an Schwefelsäure :

gefunden 39,240 pCt. berechnet $39,278 \mathrm{pC}$.

${ }^{*}$ ) Als Separatabdruck ars den Ber. d. naturw.-med. Vereins Inusbruck vom Verfasser eingesandt. 